\title{
Feature Point-Based Object Tracking
}

\author{
Xin Wang* Member \\ Masanori Sugisaka** Member \\ Wenli $\mathrm{Xu}^{* * *}$ Non-member
}

In this paper, we describe a new algorithm for tracking a known static target in image frames from a moving platform. We have noticed that a image point is environment sensitive, but those changes of grouped points energy have their own statistical similarities in two image frames within limited time interval. This approach analyzes correspondence of interest points around every feature points between inter-frames in image sequence in order to decide those feature points. We tackle these tasks with three broad approaches. First, we make an active contour model of a target in order to build some low-energy feature points. The feature points give constraints of the input state space for interest point detection in an input image frame. The second step is a method of detecting interest points around every kernel. We take into account auto-correlation method to indicate the presence of interest points for the purpose of features state space in consecutive image frames that can be tracked. The third step of our strategy is to detect the correspondence of interest points by a probabilistic relaxation method in tracking windows. The detecting process is iterative and begins with the detection of all potential correspondence pair in consecutive image. Each pair of corresponding points is then iteratively recomputed to get a globally optimum set of pairwise correspondences. Successful results are given for a real vide frames.

Keywords: image frame, feature point, correspondence

\section{Introduction}

Motion detection is made difficult as both the observer and some elements of the scene may be moving. But detecting objects from a moving platform is one of the key issues to the successful applications of mobile system, for example, mobile robot system. Zelnik and Irani ${ }^{(1)}$ presented a method for extending two-frame planar motion estimation techniques into a simultaneous multi-frame estimation, by exploiting multi-frame subspace constraints of planar surfaces. Their method allows for varying camera calibration, but some parameter vectors are needed because this method is an expansion of two-frame parametric alignment. Medioni and Cohen et $\mathrm{al}^{(2)}$. presented an event detection and analysis system. They detect and track moving region in an image frames and take as input trajectories together with user-provided information to instantiate scenarios. But in this method, a reference image from the target image sequence is necessary. Peterfreund ${ }^{(3)}$ proposed Kalman filter-based active contour model to track position and velocity. Zhong and Jain et $\mathrm{al}^{(4)}$. proposed a method for object tracking using prototype-based deformable models. Rasmussen and Hager ${ }^{(5)}$ proposed the probabilistic data association methods to improve tracking perfor-

\footnotetext{
* Dept. of Automation, Tsinghua University, Beijing, 100813, and the Dept. of Electrical and Electronic Engineering, Oita University, Oita 870-1192

** Dept. of Electrical and Electronic Engineering, Oita University, Oita 870-1192

*** Dept. of Automation, Tsinghua University, Beijing, 100813
}

mance in difficult visual environments. These researches have gotten good results under some constraints.

However, all of the above methods are weak in changing background or need reference frame or template. We present a new algorithm that track a static object by its feature points in image frames from a moving platform. We tackle these tasks with three broad approaches. First, we have augmented the general idea of snake $^{(6)}$ to make an active contour model of a target or object. This work is aimed at selecting some low-energy feature points called kernels about a target/object, giving local areas around every kernel. The kernels are defined as the final converged control points of the active contour. Those control points are also given by coarse hand initiation ${ }^{(3)(5)(12)}$. The second part is a method of detecting interest points around every kernel in an image frame. We use auto-correlation detecting technique, which is similar to the works ${ }^{(7) \sim(9)}$. The third part of our strategy is a probabilistic relaxation method for detecting the correspondence of interest points. In this algorithm, the detecting process is iterative and begins with the detection of all potential correspondence pair in consecutive image. Each pair of corresponding points is then iteratively recomputed to get a globally optimum set of pairwise correspondences. Those detected feature points are also used as the input of next image frame. The part two and part three's works are repeated. The results of part three are used to decide the correspondence interest points around the kernel in part two. If we know the corresponding points around a kernel in a new frame, we can decide the kernel by simple chain 
code $^{(11)}$. So we can track a target by understanding its kernels because the kernels are the feature points of the target.

Comparing with main existing tracking algorithm, template-based region tracking ${ }^{(2)(4)}$, color tracking ${ }^{(5)}$, our algorithm works for unknown and constantly changing backgrounds. The presented method is based on calculating the local minimum energy points around a feature point, analyzing the features of image tensor, and then deciding the corresponding relations between frame intervals. Comparing with existing active contour based method ${ }^{(3)(5)(15)}$, we only use the contour to initialize feature points and detect the corresponding converged points around the feature points instead of searching contour shape itself. Comparing with normal optical flow method ${ }^{(13)(18)}$, this method analyzes correspondence of feature points between frames in image sequence to decide feature points of a target. The interframe time interval also maybe not short enough. What is closely related to our approach is the work by ${ }^{(17)}$. One of important differences between motion in moving camera or moving platform and static camera is that all is moving in the former case and only object are moving in the later. We discuss the case of a camera on a moving platform and the target/object is static on the assumptions that motion is with maximum frame velocity constraint, which a target is scanned at discussing frame time intervals, and with target/object mutual correspondence, which the target exhibits some stable points and not all but part of these points correspond to exactly some points in the next image frame.

\section{Background}

In recent years, interest in motion processing has increased with advances in motion analysis methodology and processing capabilities. A common motion analysis problem is to obtain comprehensive information about moving and static targets/objects presented in a scene. Prior knowledge, which includes information about the camera motion, mobile or static, and information about the time interval between consecutive images for example man-aid initial selection or varies information base, can also help to decrease the complexity of analysis.

The notable algorithms of objective from a moving platform using a vision sensor camera use temporal or spatial disparities estimated from the image sequence for detection purpose. Generally, there are three groups of motion-related problems from the practical point of view: motion detection, object detection and location, and derivation of $3 \mathrm{D}$ object properties. Our problem belongs to the second group, i.e. tracking a static object/target from a moving platform. Our method is to search corresponding feature points, and to tracking them.

\section{Our Approach}

In our approach, we build a contour model of a target/object to get some low-energy feature points kernels. In order to get kernel's corresponding relation between frames, it is necessary to select relevant key ar- eas around these kernels, to find some interest points in these areas, and then to get the optimal correspondences or possible solutions.

We denote $I(\mathbf{x}),\left\{\mathbf{x}=(\mathrm{x}, \mathrm{y}): \mathbf{x} \in \mathcal{R}^{2}\right\}$, as a frame in an image sequence and the $i_{t h}(i=1,2, \ldots, n)$ frame as $I_{i}(\mathbf{x})$.

3.1 Description of Active Contour The traditional active contour model, snake, is defined as an energy minimizing spline-the snake energy depends on its shape and location within an image. The main idea of snake model is to estimate an initialized curve, and use this curve to initialize the conventional snake equations in order to get solutions about its Euler-Lagrange equation. It is recomputed to eliminate high-energy segments and renew the low-energy contours until constrains are satisfied. The energy functional is a weighted combination of internal and external forces. The internal forces emanate from the shape of the snake, while the external forces come from the image and/or from higher-level image understanding processes. The snake is defined parametrically as $\mathbf{v}=v(s, t)=[x(s, t), y(s, t)]$, where $x(s, t)$, $y(s, t)$ are $x, y$ co-ordinates along the contour for some spatial parametric domain $s \in[0,1]$ and time $t, v(s, t)$ can be approximated as a B-spline. The energy functional to be minimized may be written as:

$$
\begin{aligned}
E_{s}^{*} & =\int_{0}^{1} E_{\text {sna }}(\mathbf{v}) d s \ldots \ldots \ldots \ldots \ldots \ldots \ldots \ldots \ldots \ldots \\
& =\int_{0}^{1}\left(E_{\text {int }}(\mathbf{v})+E_{\text {ext }}(\mathbf{v})\right) d s \ldots \ldots \ldots \ldots \ldots
\end{aligned}
$$

where $E_{\text {int }}(\mathbf{v})$ represents the internal energy of the spline due to bending. External energy $E_{\text {ext }}(\mathbf{v})$ includes image forces $E_{i m}(\mathbf{v})$ and external constraint forces $E_{\text {cst }}(\mathbf{v})$ :

$$
E_{\text {ext }}(\mathbf{v})=E_{i m}(\mathbf{v})+E_{c s t}(\mathbf{v}) \cdots \cdots \cdots \cdots \cdots
$$

$E_{i m}(\mathbf{v})$ is derived from the image data over which the snake lies. $E_{i m}(\mathbf{v})$ is defined as

$$
E_{i m}(\mathbf{v})=-|\nabla I(\mathbf{x})|^{2}
$$

where $I(\mathbf{x})$ denotes image gray-levels at image location $\mathbf{x}$. $E_{c s t}(\mathbf{v})$ come from external constraints imposed either by a user or some other higher-level process which may force the snake toward or away from particular features. Not loss common, we just select $E_{c s t}(\mathbf{v})=0$ for computation simplified.

From (2), the functional to be minimized is (1). Then from the calculus of variations, the Euler-Lagrange condition states that the spline curve $v(s, t)$ which minimizes $E_{\text {sna }}(\mathbf{v})$ must satisfy

$$
\frac{d}{d s} E_{v_{s}}-E_{\mathbf{v}}=0 \ldots \ldots \ldots \ldots \ldots \ldots \ldots \ldots
$$

where $E_{v_{s}}$ is the partial derivative if $E(\mathbf{v})$ with respect to $d v(s) / d s$ and $E_{\mathbf{v}}$ is the partial derivative of $E_{s n a}(\mathbf{v})$ with respect to $\mathbf{v}$. Then the Euler-Lagrange equation reduces to 


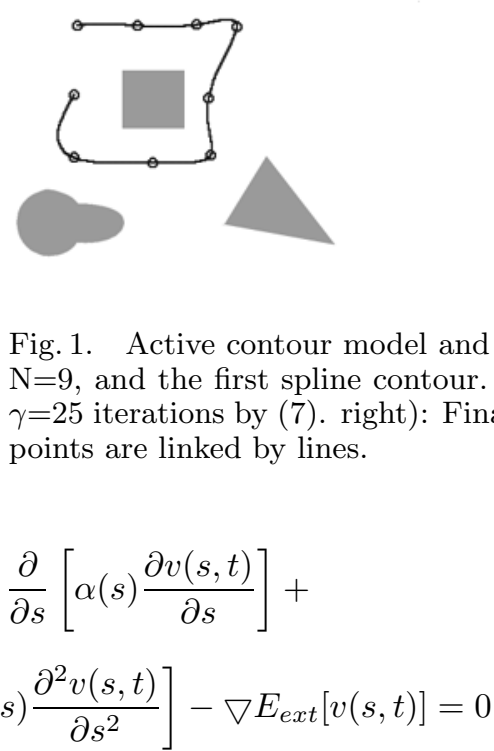

where $\alpha(s), \beta(s)$ specify the elasticity and stiffness of the snake.

We give a snake contour with $N$ nodes, $n_{i}=\left(u_{i}, w_{i}\right)$, $i=1,2, \ldots, N$, the state of the snake is represented by $(\mathbf{u}, \mathbf{w})=\left(\left(u_{1}, \ldots, u_{N}\right)^{T},\left(w_{1}, \ldots, w_{N}\right)^{T}\right)$. The resolution above the Euler-Lagrange equation (6) can then be written as:

$$
\mathbf{A Z}+\gamma \mathbf{\Delta} \mathbf{Z}+\mathbf{\Sigma} \mathbf{F}=\mathbf{0} \ldots \ldots \ldots \ldots \ldots \ldots
$$

where $\mathbf{A}=\mathbf{A}(\alpha, \beta)$ is a pentadiagonal banded matrix that depends on $\alpha$ and $\beta$ which control the internal spline energy of the snake. $\mathbf{Z}=\mathbf{Z}(\mathbf{u}, \mathbf{w}), \mathbf{F}$ denotes image external force. We define $\mathbf{F}_{i m}=E_{i m}(\mathbf{v})$, and expend the snake with a balloon force as $\mathbf{F}_{b}, \mathbf{F}_{b}=E_{c s t}(\mathbf{v})$, then $\boldsymbol{\Sigma} \mathbf{F}=\mathbf{F}_{b}+\mathbf{F}_{i m}$. After $\gamma$ iteration steps, we can get those new points $\left(\mathbf{u}_{t}, \mathbf{w}_{t}\right)$ from $\left(\mathbf{u}_{t-1}, \mathbf{w}_{t-1}\right)$. Fig. 1 shows the process and one of our results by the described model: left): the nine initial control points are selected manually and the first contour is formed; middle): the results are given after 25 iterations; right): nine kernels are decided by the converged control points by the result in the middle figure.

3.2 Detecting Points of Interest View-based recognition from local information relies on interest points, which represent specific places in an image that carry distinctive features of the object being studied. In order to track the kernels decided in Section 3.1, we detect interest points around every kernels.

3.2.1 Contour Code We select tracking windows with different scales in input space $A_{m}^{\prime} \subset I_{i}(\mathbf{x})$ and output space $A_{n}^{\prime} \subset I_{j}(\mathbf{x})$ for the kernel's geometric characteristics. We make the tracking windows around every selected kernel $\mathbf{x}$. If more than two of elements of $A_{m}^{\prime}$ in $A_{n}^{\prime}$ are decided, the corresponding position of the kernel in $I_{j}(\mathbf{x})$ can be estimated. If some corresponding points of the kernels are found in $A_{n}^{\prime}$, we can also estimate the target in this new frame by parts of its feture points. Corresponding elements in $A_{m}^{\prime}$ and $A_{n}^{\prime}$ are recognized by simple directive chain $_{\text {codes }}{ }^{(11)}$ with their

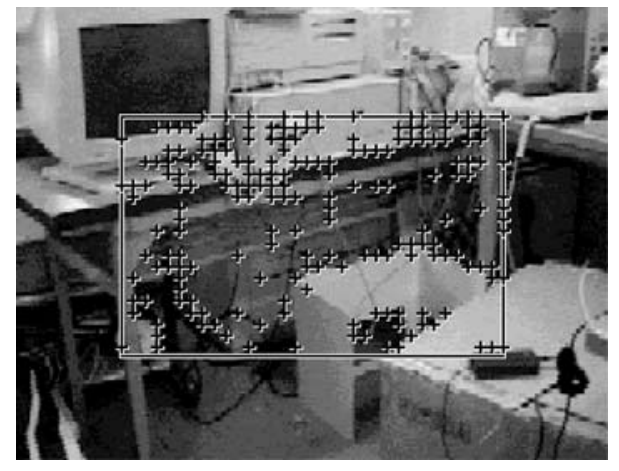

Fig. 2. Interest points of an selected image area.

neighborhoods.

3.2.2 Definition of the Transform We build up a transform relation $C(\mathbf{x})$ in a local window about a point $\mathbf{x}$ as follows:

$$
C(\mathbf{x})=\Re(\mathbf{x}) * *\left[\begin{array}{cc}
I_{x}^{2} & I_{x} I_{y} \\
I_{x} I_{y} & I_{y}^{2}
\end{array}\right] \ldots \ldots \ldots \ldots
$$

where $I=I(\mathbf{x})$ is intensity function of the image, $* *$ is the convolution operation, and $I_{x}=G_{x} * * I(\mathbf{x}), G_{x}$ is $x$-derivative of a Gaussian with standard deviation one, and similarly for $I_{y} . \Re(\mathbf{x})$ is a weight mask to weight the derivatives over the window. This matrix captures the local structure. The eigenvectors of this matrix are the principal curvatures of the auto-correlation function. Its rank one indicates an edge and rank zero a homogeneous region. Two significant values indicate the presence of an interest point. We consider a cost function $R(\mathbf{x})$ :

$$
R(\mathbf{x})=k_{1} \operatorname{det}[C(\mathbf{x})]-k_{2} \operatorname{trace}[C(\mathbf{x})]^{2} \ldots \ldots \ldots
$$

where $k_{1}$ is a scale of $I(\mathbf{x}), k_{2}$ is an experimental constant. The interest points are decided by selecting a threshold about $R(\mathbf{x})$. Fig. 2 shows one of the detected results for an image by this method. The feature points of targets/objects are limited in a window drawn by lines in Fig. 2. The detected interest points are marked by "+" symbol with white background. More results are given in Section 5 where detections are done in $A_{m}^{\prime}$ and $A_{n}^{\prime}$ respectively.

3.3 Detecting Corresponding Points After the process of interest point detection, we have to decide the relation of those interest points between $A_{m}^{\prime}$ 
and $A_{n}^{\prime}$ because the interest points cannot tell us anything about their correspondences. Let $A_{m}=\left\{\mathbf{x}_{m}\right\}$ be the set of all interest points in the first starting image that is input state space, and $A_{n}=\left\{\mathbf{y}_{n}\right\}$ the interest points in the second image that is output state space. Let $\mathbf{c}_{m n}$ be a vector connecting points $\mathbf{x}_{m}$ and $\mathbf{y}_{n}$ (thus $\left.\mathbf{y}_{n}=\mathbf{x}_{m}+\mathbf{c}_{m n}\right)$. Let the probability of correspondences of two points $\mathbf{x}_{m}$ and $\mathbf{y}_{n}$ be $P_{m n}$. Two points $\mathbf{x}_{m}$ and $\mathbf{y}_{n}$ can be considered potentially corresponding if their distance satisfies the assumption of maximum velocity,

$$
\left|\mathbf{x}_{m}-\mathbf{y}_{n}\right| \leq D_{\max }
$$

where $D_{\max }$ is the maximum distance a point may move in the time interval between two consecutive images. Two correspondences of points $\mathbf{x}_{m} \mathbf{y}_{n}$ and $\mathbf{x}_{k} \mathbf{y}_{l}$ are termed consistent of

$$
\left|\mathbf{c}_{m n}-\mathbf{c}_{k l}\right| \leq D_{d i f}
$$

where $D_{d i f}$ is a pixel distance deviation. Consistency of corresponding point pairs will increases the probability that a correspondence pair is correct. We Determine the sets of interest points $A_{m} \subset A_{m}^{\prime} \subset I_{i}(\mathbf{x})$, $A_{n} \subset A_{n}^{\prime} \subset I_{j}(\mathbf{x})$, and construct a data structure as follows:

$$
\left[\mathbf{x}_{m},\left(\mathbf{c}_{m_{1}}, P_{m_{1}}\right),\left(\mathbf{c}_{m_{2}}, P_{m_{2}}\right), \ldots,\left(N o V^{*}, N o P^{*}\right)\right]
$$

where $P_{m n}$ is the probability of correspondence of points $\mathbf{x}_{m}$ and $\mathbf{y}_{n}, N o V^{*}$, and $N o P^{*}$ are special symbols indicating that no potential correspondence was found.

We initialize the probabilities $P_{m n}^{(0)}$ of correspondence based on local similarity -if two points correspond, their neighborhood should correspond as well:

$$
P_{m n}^{(0)}=\frac{1}{1+k_{p} \omega_{m n}}\left(1-P_{\left(N o V^{*}, N o P^{*}\right)}^{(0)}\right) \cdots \cdots
$$

where $P_{\left(N o V^{*}, N o P^{*}\right)}^{(0)}$ is the initialized probability of no correspondence, $k_{p}$ is a constant and

$$
\omega_{m n}=\sum_{\boldsymbol{\Delta} \mathbf{x}}\left[I_{m}\left(\mathbf{x}_{m} \pm \mathbf{\Delta} \mathbf{x}\right)-I_{n}\left(\mathbf{y}_{n} \pm \boldsymbol{\Delta} \mathbf{x}\right)\right]^{2}
$$

$\Delta \mathbf{x}$ defines a neighborhood for image match testing -a neighborhood consists of all points $(\mathbf{x}+\Delta \mathrm{x})$, where $\Delta \mathbf{x}$ is defined as a symmetric neighborhood around $\mathbf{x}$.

We iteratively determine the probability of correspondence of a point $\mathbf{x}_{m}$ with all potential points $\mathbf{y}_{n}$ as a weighted sum of probabilities of correspondence of all consistent pairs $\mathbf{x}_{k} \mathbf{y}_{l}, \mathbf{x}_{k}$ are neighbors of $\mathbf{x}_{m}$ and the consistency of $\mathbf{x}_{k} \mathbf{y}_{l}$ is evaluated according to $\mathbf{x}_{m}, \mathbf{y}_{n}$. A quality $q_{m n}$ of the correspondence pair is defined as

$$
q_{m n}^{(s-1)}=\sum_{k} \sum_{l} P_{k l}^{(s-1)}
$$

where $s$ denotes an iteration step, $k$ refers to all points $\mathbf{x}_{k}$ that are neighbors of $\mathbf{x}_{m}$, and $l$ refers to all points $\mathbf{y}_{l} \in A_{n}$ that form pairs $\mathbf{x}_{k} \mathbf{y}_{l}$ consistent with the pair

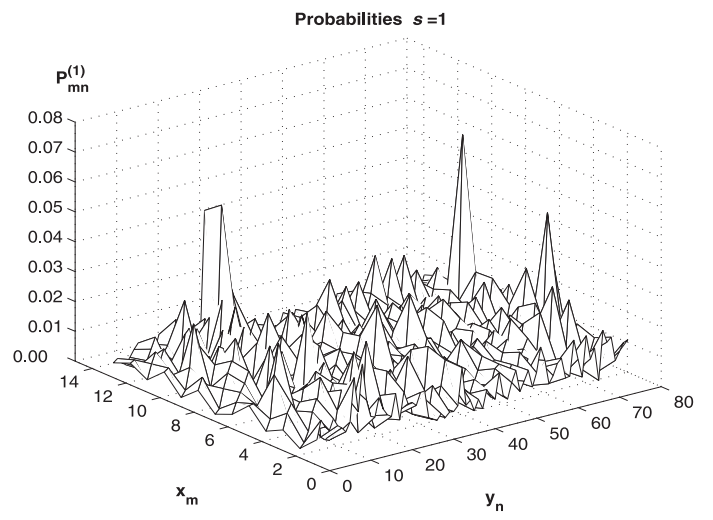

Fig. 3. Interest point's probability distribution when iteration step $s=1$.

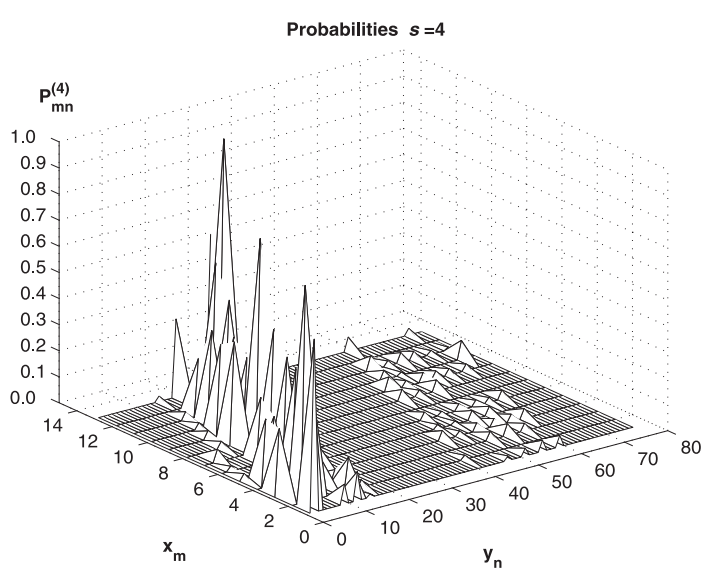

Fig. 4. Interest point's probability distribution when iteration step $\mathrm{s}=4$.

$\mathbf{x}_{m} \mathbf{y}_{n}$

The probabilities of correspondence are updated for each point pair $\mathbf{x}_{m}, \mathbf{y}_{n}$.

$$
\hat{P}_{m n}^{(s)}=P_{m n}^{(s-1)}\left(k_{a}+k_{b} q_{m n}^{(s-1)}\right)
$$

where $k_{a}$ and $k_{b}$ are preset constants. They deal with the convergent speed of $P_{m n}$. Normalize

$$
P_{m n}^{(s)}=\frac{\hat{P}_{m n}^{(s)}}{\sum_{j} \hat{P}_{m j}^{(s)}}
$$

Those interest points that hold high probabilities that obviously differ from those interest points without correspondences. Repeat (15) (16) and (17) until the $P_{m n}^{(s)}>P_{t h r}$ (threshold) is found for all points $\mathbf{x}_{m}, \mathbf{y}_{n}$.

Fig. 3 - Fig. 5 illustrated some results in our experiments (Also see Section 5). There are 13 and 76 elements in $A_{m}$ and $A_{n}$ respectively (Fig. 3). 13 interest point pairs are detected after $s=10$ iterations (Fig. 5). The first $P_{m n}^{(1)}(s=1)$ and the fourth $P_{m n}^{(4)}(s=4)$ are illustrated in Fig. 3 and Fig. 4. Details are also illustrated in Section 5.

\section{The Measurement Process}

Two target/object frames $I_{i}(\mathbf{x}), I_{j}(\mathbf{x})$, in which the 
target is, are sampled by their appeared orders in image sequence. The input frame is $I_{i}(\mathbf{x})$, output is $I_{j}(\mathbf{x})$. In-

Probabilities $s=10$

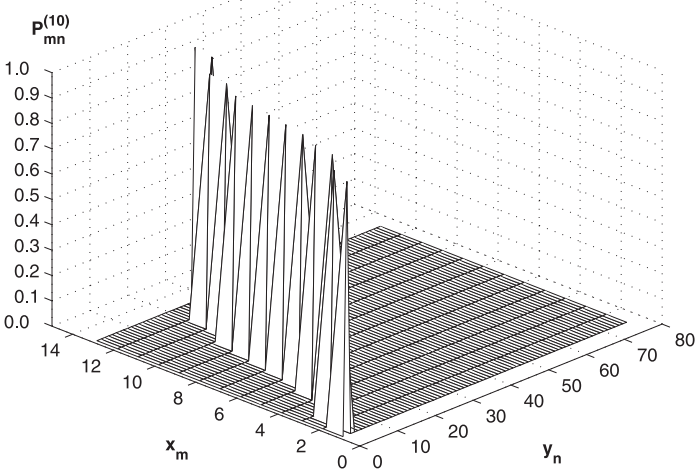

Fig. 5. Interest point's probability distribution when iteration step $\mathrm{s}=10$. tuitively, we give $N$ local approximate location in $I_{i}(\mathbf{x})$ by coarse hand initialization. After executing the algorithm described in Section 3.1, an active contour model is formed first and then the contour converges to $N$ low-energy positions. Consequently, the kernel state space $\breve{A}_{m}$ are those feature points with low-energy state. There are $N$ kernels in the state space $\breve{A}_{m}$. An input state space $A_{m}$ (see Section 3.2 ), $A_{m}=\left\{\mathbf{x}_{m}\right\}$, is constructed around a kernel in $\breve{A}_{m}$. $A_{m}^{\prime}$ gives a tracking window in $I_{i}(\mathbf{x})$. We can compute the possible interest points around every kernel in the tracking window, and build another tracking window $A_{n}^{\prime}$ including a corresponding output state space $A_{n}=\left\{\mathbf{y}_{n}\right\}$ in $I_{j}(\mathbf{x})$. In this paper, we give the results of one kernel between frames to illustrate our method. This principle is also suitable for more kernels. The area of $A_{n}^{\prime}$ is up to the maximum distance $D_{\max }$ in (10). Finally the corresponding relations are established by application of the algorithm presented in Section 3.3. A kernel's position will be de-
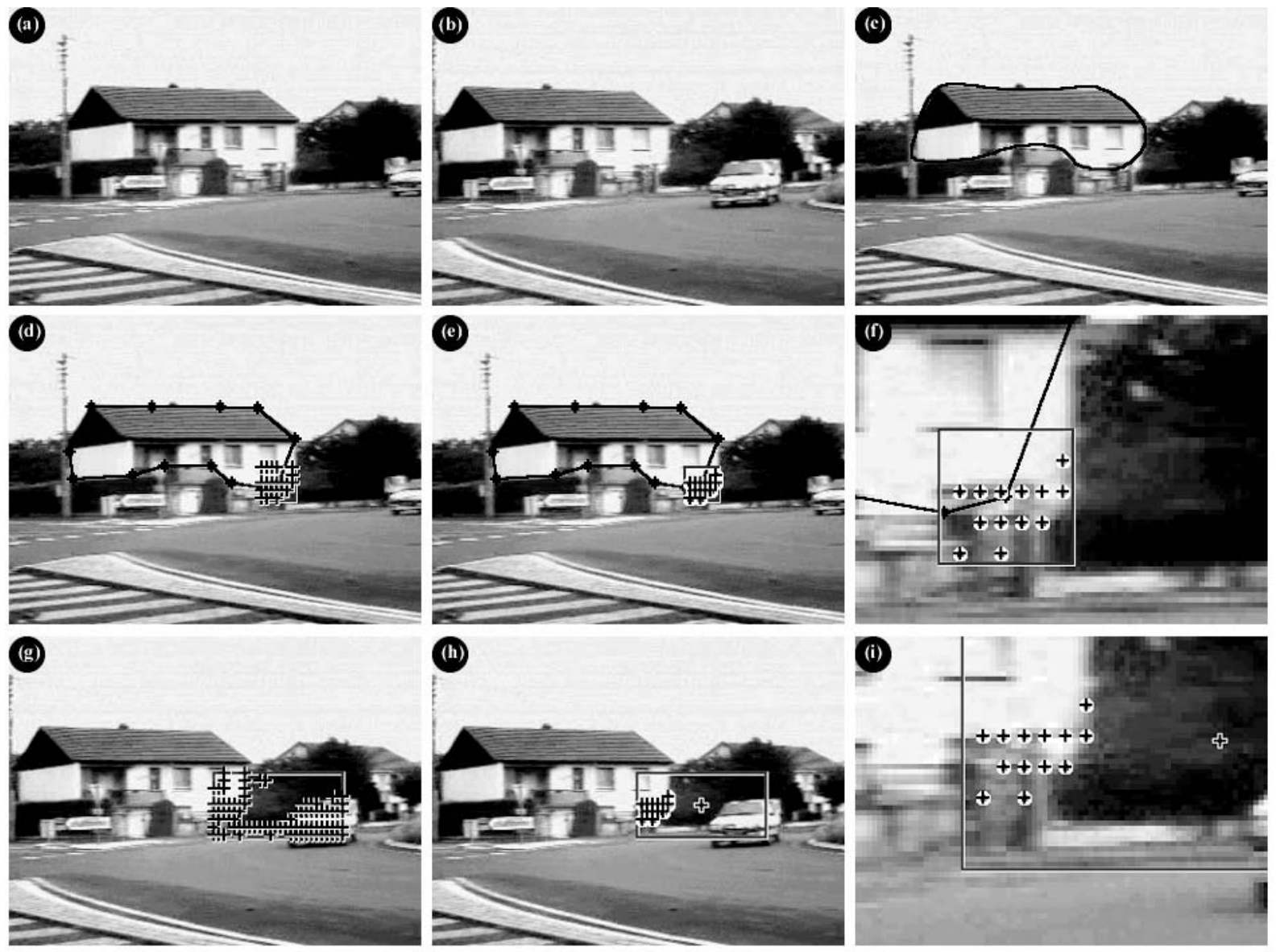

Fig. 6. Tracking a house corner in image sequence from a moving platform. (a): An original input frame that is the second frame in an image sequence. (b): The original corresponding output frame which is the ninth frame in the same image sequence as (a). (c): The converged spline contour model. (d): The kernels of the house, which are linked by lines. The original interest points after the first detection in a local area are marked by "+". (e): The corresponding points of (d) with the threshold and constraints, are marked by "+" symbols with white circle background. (f): Significant part concerning with correspondent points in (e) is amplified. (g): The interest points in a larger area in the frame 9 are marked by "+" symbols with white circle background. (h): The corresponding points of $(\mathrm{g})$. The kernel/s position is marked by black-white "+" symbols. (i): Significant part concerning with correspondent points in (h) is amplified. 
scribed by the points coding chain gotten in the input state space. We get a new kernel In the frame $A_{n}^{\prime}$ including $A_{n}=\left\{\mathbf{y}_{n}\right\}$. More kernels, which are to form a new input space state, can also be calculated by repeating the same process in Section 3.2 and Section 3.3. The target/object is tracked by tracking its these kernels in consecutive image frames. This process is illustrated in (18).

$$
\begin{aligned}
\breve{A}_{m}^{(t)} \Rightarrow & A_{m}^{\left(t_{1}\right)}, A_{n}^{\left(t_{1}\right)} \Rightarrow \breve{A}_{m}^{\left(t_{1}\right)} \Rightarrow A_{m}^{\left(t_{2}\right)}, A_{n}^{\left(t_{2}\right)} \\
\ldots \Rightarrow & \breve{A}_{m}^{\left(t_{n}\right)} \Rightarrow A_{m}^{\left(t_{n+1}\right)}, A_{n}^{\left(t_{n+1}\right)} \ldots \\
& \ldots \ldots \ldots \ldots \ldots \ldots \ldots \ldots \ldots \ldots \ldots \ldots \ldots
\end{aligned}
$$

where $t_{k}, k=1,2, \ldots$, denotes sampling time at $k$. The sampling interval relies on the velocity of relative moving platform.

\section{Experimental Results}

In this section, some experimental results based on the presented method are illustrated. We use the same video image [1] to execute our experiments. The second and the ninth frames are selected in this experiment. In Fig. 6, (a) is the original input image frame 2; (b) is the output images frame 9 ; (c) gives the active contour model based on the algorithm described in Section 3.1. The parameters for this model are $N=12, \alpha=0.6$, $\beta=0.0, \gamma=25, \mathbf{F}_{i m}=-|\nabla I(\mathbf{x})|^{2}, \mathbf{F}_{b}=0$; The kernels and an area of $A_{m}^{\prime}$ are shown in (d). $A_{m}^{\prime} \subset R^{2}$, there are $24 \times 24$ (unit: 5 pixels) elements in it. To show the process of (8) and (9) in Section 3.2, (d) gives the 21 original interest points after the first detection. The parameters in (9) are $k_{1}=256, k_{2}=0.06$. 13 of the 21 interest points are then kept after excluding the points on the edge; (g) shows the interest points in $A_{n}^{\prime}, A_{n}^{\prime}$ $\subset R^{2}$, there are $100 \times 50$ elements in it. $A_{m}$ and $A_{n}$ are detected from $A_{m}^{\prime}$ and $A_{n}^{\prime}$ respectively, $A_{m} \subset A_{m}^{\prime}, A_{n}$ $\subset A_{n}^{\prime} . A_{n}$ is in a larger area so there are more elements i.e. interest points in it. The reason why a larger $A_{n}^{\prime}$ in (g) is selected is that the size of $A_{n}^{\prime}$ up to the velocity constraints of $D_{\max }$ in (10) resulted from the moving camera. The larger the size is the more interest points are detected, but also accompanied with much time cost. (e) and (h) give the detected results by using the algorithm described in Section 3.3. The kernel's position is also marked by black-white "+" where is the center of mass in $A_{n}^{\prime}$. $\mathbf{x}_{m}$ and $\mathbf{y}_{n}$ are located by "+" symbols with white circle background in (e) or (h). All 13 pairs of elements are decided between $A_{m}$ and $A_{n}$. (e) and (h) show that even if we selected a smaller local area around a kernel, it is still possible to catch some corresponding elements in $A_{n}$ since kernels locate on the low energy positions. To show these results more clearly, (f) and (i) focus on the detected corresponding part in (e) and (h), and give their amplified effects. The results have given very good corresponding relations, and they are also presented in Fig. 3 - Fig. 5 where the values of $\mathbf{x}_{m}$ and $\mathbf{y}_{n}$ are their indexes in $A_{m}$ and $A_{n}$ in their storage spaces.

We select pixel distance in a time interval $D_{\max }=37.5$ pixels, $D_{\text {dif }}=1$ pixel, $s=10, P_{t h r}=0.70$. The corre- spondence points are decided with errors less than 5 pixels because our calculating unit is 5 pixels. The results have been good enough to analysis the motion features and to be as a kernel for the next step.

\section{Conclusions}

This paper's primary contribution is its demonstration of a method of feature points-based motion analysis. It realizes tracking a static known target/object in image frames taken from a moving camera. We proposed a new approach starting from some converged points by the snake principle. We depict the attributes of a target by its significant energy points. Our idea to understand a target is from its points of this target to its lines, from its points to its lines and areas. This is related to manipulate our knowledge database in our further works. The proposed interest point detection approach also gives very satisfied results.

In this work, we proposed three processes to track a static target from a moving platform where an camera is moving and an target is static: active contour model, which are used to estimate kernel points at the final converged contour position; the interest point detection, which is in a area around kernel, along with measures of auto-correlation transform, as the system input measurement; and a probabilistic relaxation method, which uses correspondence pairs detection, as the former system output and the next system input. This approach takes us another advantage to occlusion problems, because the target/object can be estimated by parts of their features.

In future work, we hope to rationalize the selection of visual cues used for target/object tracking automatically. Utilizing image deformable models is one of possible ways. Another interesting question is how to efficiently apply the described method to autonomous car/robot navigation.

(Manuscript received July 22, 2003, revised Jan. 14, 2004)

\section{References}

(1) L. Zelnik and M. Irani: "Multi-frame estimation of planar motion", IEEE Trans. Pattern Analysis and Machine Intelligence, Vol.22, No.10, pp.1105-1116 (2000)

( 2 ) G. Medioni, I. Cohen, F. Bremond, S. Hongeng, and R. Nevatia: "Event detection and analysis from video streams", IEEE Trans. Pattern Analysis and Machine Intelligence, Vol.23, No.8, pp.873-889 (2001)

( 3 ) N. Peterfreund: "Robust tracking of position and velocity with Kalman snakes", IEEE Trans. Pattern Analysis and Machine Intelligence, Vol.21, No.6, pp.564-569 (1999)

(4) Y. Zhong, A. K. Jain, M. Dubisson, and P. Jolly: "Object tracking using deformable templates", IEEE Trans. Pattern Analysis and Machine Intelligence, Vol.22, No.5, pp.544-549 (2000)

( 5 ) C. Rasmussen and G. D. Hager: "Probabilistic data association methods for tracking complex visual object", IEEE Trans. Pattern Analysis and Machine Intelligence, Vol.23, No.6, pp.560-576 (2001)

(6) M. Kass, A. Witkin, and D. Terzopouls: "Snakes: active contour models", Int'l J. Computer Vision, Vol.1, No.4, pp.321331 (1988) 
( 7 ) L.D. Cohen and I. Cohen: "Finite-element methods for active contour models and balloons for 2-D and 3-D images", IEEE Trans. Pattern Analysis and Machine Intelligence, Vol.15, No.11, pp.1131-1147 (1993)

(8) C. Schmid and R. Morh: "Local gray value invariants for image retrieval", IEEE Trans. Pattern Analysis and Machine Intelligence, Vol.19, No.5, pp.530-535 (1997)

( 9 ) C. Schmid, R. Morh, and C. Bauckhage: "Evaluation of interest point detector", Int'l J. Computer Vision, Vol.37, No.2, pp.151-172 (2000)

(10) G. Carneiro and A. Jepson: "Phase-based local features", Computer Vision - ECCV 2002, Part I, pp.283-296 (2002)

(11) B. Jähne: "Iamge Processing for Scientic Application", CRC Press, New York (1997)

(12) J. MacCormick: "Stochastic algorithms for visual tracking", Springer-Verlag, London (1998)

(13) A. Kumar, A. Tannenbaum, and G. Balas: "Optical flow: a curve evolution approach", IEEE Trans. Image Proc., Vol.5, No.5, pp.598-610 (1996)

(14) D. Nair and J. K. Aggarwal: "Moving obstacle detection from a navigation robot", IEEE Trans. Robotics and Automation, Vol.14, No.3, pp.404-416 (1998)

(15) N. Paragios and R. Deriche: "Geodesic active contours and level sets for the detection and tracking of moving objects", IEEE Trans. Pattern Analysis and Machine Intelligence, Vol.22, No.3, pp.266-280 (2000)

(16) S. Sclaroof and L. Liu: "Deformable shape detection and description via model-based region grouping", IEEE Trans. Pattern Analysis and Machine Intelligence, Vol.23, No.5, pp.475-489 (2001)

(17) L. Torresani and G. C. Bregler: "Space-Time Tracking", Computer Vision - ECCV 2002, Part I, pp.801-812 (2002)

(18) R. Hummel and V. Sundareswaran: "Motion parameter estimation from global optical flow data", IEEE Trans. Pattern Analysis and Machine Intelligence, Vol.15, No.5, pp.459-476 (1993)

(19) D. Kottke and Y. Sun: "Motion estimation via cluster matching", IEEE Trans. Pattern Analysis and Machine Intelligence, Vol.16, No.11, pp.1128-1132 (1994)

(20) W. Yu, K. Daniilidis, and G. Sommer: "Approximate orientation steer ability based on angular Gaussians", IEEE Trans. Image Proc., Vol.10, No.2, pp.193-205 (2001)
Xin Wang (Member) received the $\mathrm{PhD}$ degree in electrical and electronic engineering from Oita University in 1998. From 1998 to 1999, he was a visiting researcher at Oita University. From 1999 to 2003, he has been with Department of Electronics at Oita Institute of Technology. Since 2003, he has been a part-time lecturer at Oita University and a postdoctoral researcher at Tsinghua University (Beijing). He is currently employed with the Graduate School of Engineering, University of Tokushima. His current research interests include computer vision and image processing with their practical applications, vision-based control, autonomous robot navigation, and autonomous control for welfare services. He is a member of the Institute of Electrical Engineers of Japan (IEEJ) and a member of the IEEE.

Masanori Sugisaka (Member) received the $\mathrm{PhD}$ degree in

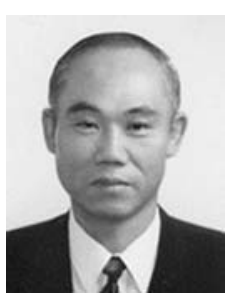
electrical engineering from Kyushu University in 1975. From 1973 to 1974 , he was a research associate at Oita University. Since 1988, he has been a professor at Oita University. Now he belongs to the Graduate School of Electrical and Electronic Engineering, Oita University. His current research interests are in the fields of artificial life and robotics, especially artificial brain. He is a member of the Institute of Electrical Engineers of Japan, a member of SICE and a member of the IEEE.

Wenli Xu (Non-member) was born in 1947. He received the

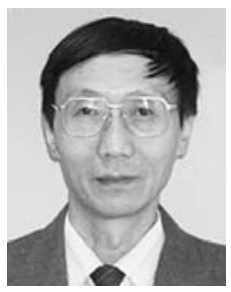
B.S. degree in electrical engineering and the M.E. degree in automatic control engineering from Tsinghua University, Beijing, China, in 1970 and 1980, respectively, the PhD degree in electrical and computer engineering from the University of Colorado at Boulder, $\mathrm{CO}$, in 1990. He is currently a professor of Tsinghua University and a director of Chinese Association of Automation. His research interests are mainly in the areas of automatic control and computer vision. 